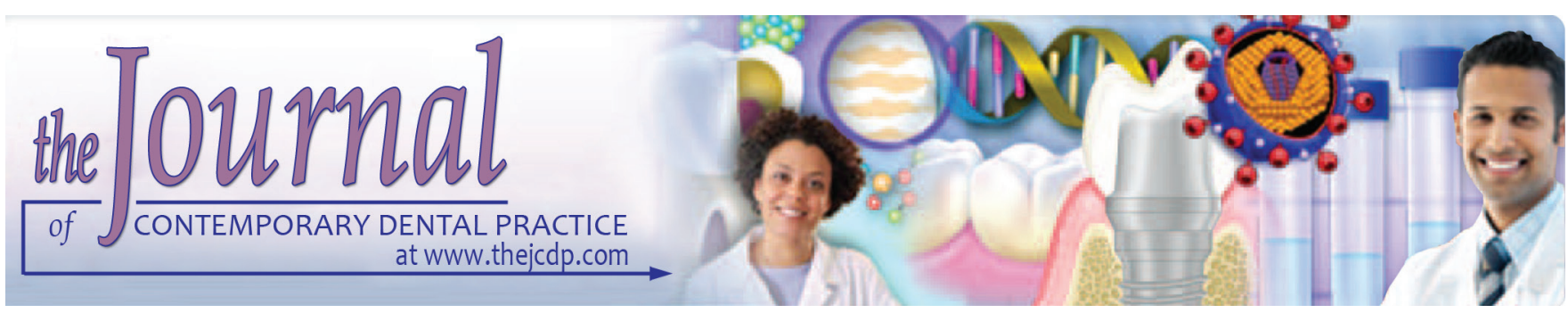

\title{
Comparison of Shear Bond Strength and Microleakage of Various Bulk-fill Bioactive Dentin substitutes: An in vitro Study
}

${ }^{1}$ Fahad I Alkhudhairy, ${ }^{2}$ Zeeshan H Ahmad

\begin{abstract}
Introduction: Various bulk-fill materials depending on their composition, viscosity, and flow ability have different physical and mechanical properties. The aim of this in vitro study was to determine and compare the shear bond strength and microleakage properties of activa restorative with other bulk-fill restorative materials surefil (SDR), Biodentine, ever X posterior.

Materials and methods: Forty permanent premolars were selected for shear bond strength, and 20 permanent premolars were selected with class II cavities on mesial and distal side for microleakage. Universal testing device was used to assess the shear bond strength. Microleakage was checked using dye penetration method under a stereomicroscope. Mean and standard deviation values were calculated from the recorded values. Intergroup comparison was done by one-way analysis of variance (ANOVA) followed by pairwise comparison using Tukey honestly significant difference (HSD) post hoc test.
\end{abstract}

Results: The mean shear bond strength was highest for SDR surefil followed by Ever $\mathrm{X}$ posterior, Bioactive restorative, and Biodentine respectively. In this study, SDR (surefil) showed better shear bond strength and better microleakage properties compared with the other test materials $(F=186.7157, p<0.05)$.

Conclusion: The result of this study showed that flowable and fiber-reinforced composites have better shear bond strength and microleakage properties.

Clinical significance: Flowable bulk-fill composite resins can be used as dentin substitutes because of its superior properties.

Keywords: Bulk-fill, Dentin substitute, Microleakage, Shear bond strength.

${ }^{1}$ Department of Restorative Dental Sciences, King Saud University, College of Dentistry, Riyadh, Kingdom of Saudi Arabia

2Department of Endodontics, College of Dentistry, King Saud University, Riyadh, Kingdom of Saudi Arabia

Corresponding Author: Zeeshan H Ahmad, Department of Endodontics, College of Dentistry, King Saud University, Riyadh Kingdom of Saudi Arabia, e-mail: zeeshan-a-h@yahoo.com
How to cite this article: Alkhudhairy Fl, Ahmad ZH. Comparison of Shear Bond Strength and Microleakage of Various Bulk-fill Bioactive Dentin substitutes: An in vitro study. J Contemp Dent Pract 2016;17(12):997-1002.

Source of support: Nil

Conflict of interest: None

\section{INTRODUCTION}

The traditional way of cavity preparation was material driven and involved tooth destruction. Recent advancement in adhesive dentistry has restricted the cavity size and shape to minimally invasive. ${ }^{1}$ Recent advances in the restorative materials and their properties have given clinicians wide options to choose different clinical situations. ${ }^{2}$ Finding the appropriate posterior restorative material in stress-bearing areas is still a challenge, as bulk fracture is considered one of the main reasons for restoration failure. ${ }^{3}$ Bulk-fill composites were introduced in an effort to improve the performance of composite resin restoration mainly in the posterior areas to have good physical and mechanical property to endure high masticatory stresses. Bulk-fill composites reduce the chair-side time and have less risk of air entrapment and moisture contamination. ${ }^{4}$

Various bulk-fill materials depending on their composition, viscosity, and flow ability have different physical and mechanical properties. Advances led to the addition of fiber in normal conventional dental composites to enhance their physical and mechanical properties, so that the stress transfer from the matrix to the fiber depends on the fiber length and diameter to resist fracture of restoration. ${ }^{5}$ To overcome shortcomings of direct esthetic posterior restorative materials, such as operator dependence, the need for a bonding system, and poor biocompatibility, a tricalcium silicate-based cement was introduced. This 
new material was designed as dentin substitute and for direct posterior restorations.

Biodentine material other than being biocompatible is also bioactive, which can stimulate odontoblast differentiation from pulp progenitor cells for regeneration of dentin. The handling properties of the material and its mechanical behavior make it a preferred choice as dentin substitute in direct posterior restoration. The material exhibits good dimensional stability and gives a good marginal fit for restoration. ${ }^{6}$

The one of its kind bioactive dental material with ionic resin matrix is Activa Restorative. It possesses the shockabsorbing resin component, bioactive fillers, similar to the properties of natural teeth. These restorations are durable, fracture and wear resistant, show chemical binding to the teeth, show less microleakage, and help in release and recharge of calcium, phosphate, and fluoride ions from glass ionomers. They are free from bisphenol A derivatives. ${ }^{7}$

Thus, the aim of this in vitro study was to determine and compare the shear bond strength and microleakage properties of activa restorative with other bulk-fill restorative materials surefil (SDR), biodentine, and Ever $\mathrm{X}$ posterior.

\section{MATERIALS AND METHODS}

An in vitro study was conducted to compare the shear bond strength and miroleakage properties of four bulk-fill restorative materials namely activa bioactive restorative, biodentine, everx posterior, and SDR (surefil, Dentsply). Sample size was calculated from previously available literature with $95 \%$ confidence interval and $80 \%$ power of the study. Ethical clearance was obtained from the Institutional Review Board. Sixty human permanent noncarious premolars were collected, and surface debridement was done with ultrasonic scaler and were stored in distilled water at room temperature till the experiment period. Forty permanent premolars were selected for shear bond strength and 20 permanent premolars were selected with class II cavities on mesial and distal side for microleakage.

\section{Shear Bond Strength}

Forty permanent premolars were divided into four groups and horizontal indentations were made on radicular portion. The cold cure acrylic resin molds were made with the root portion of each tooth embedded and crown portion exposed and parallel to the base.

A high-speed fine diamond disk (Isomet 2000 Precision saw, Buehler USA) with copious water spray was used to expose mid-coronal dentin of the occlusal surface, cut perpendicular to the long axis of the tooth.
Subsequently 400 grit aluminum oxide (Automata Grinding, Jeanwirtz, GmbH, Germany) abrasive paper was used to obtain flat dentin surface. Specimens were then stored in distilled water at room temperature. All samples were rinsed with distilled water and air dried directly before the application of the test material.

An impression was taken from polyether rubber impression material (Express STD; 3 M ESPE, Dental Products, MN, USA) of a columnar metal blank with a diameter of $4 \mathrm{~mm}$ and height of $6 \mathrm{~mm}$. Standardization of samples was done using these negative forms. The polyether rubber mold was placed on the dentin samples and was filled with the test material. Care was taken to avoid any air entrapments, voids, or gaps. The crosssectional area of the specimen and the test material had complete contact to the dentin surface without touching the enamel. Activa Bioactive Restorative, Biodentine, EverX Posterior, and SDR (surefil, Dentsply) were strictly handled in accordance with the manufacturer's instructions. For biodentine, no surface treatment was done for the dentin before application. After placing the mold on the dentin surface, biodentine was completely filled into the mold with the aid of cement plugger. In SDR (Surefil, Dentsply), the dentin surface was acid treated (37\% phosphoric acid) and then conditioned with Tetric N Bond (Ivoclar Vivadent) after light curing (Bluephase G2, Ivoclar Vivadent), the surefil composite resin was put in the mold and light cured in increments. For EverX posterior (GC Europe), the dentin surface was conditioned by one-step self-etch with G-aenial Bond (GC Europe), the EverX posterior composite resin was put in the mold with the aid of a cement plugger and light cured. Activa Bioactive Restorative (Pulpdent, USA) was applied after conditioning the dentin surface with $37 \%$ phosphoric acid.

Ten specimens ( $n=10$ ) were produced for every test material for shear bond testing and stored in an incubator (Memmert Universal Oven, Germany) at $37.5^{\circ} \mathrm{C}$ and $100 \%$ humidity for 2 days. The molds were removed carefully from the specimens so that only the cylindrical test material remained adhered perpendicular to the dentin surface.

The shear bond strength was evaluated using universal testing device (Instron 5965, USA). The specimens were mounted in a metal mold which served as drive surface for a metal plunger. This plunger touched the cylindrical test material at the contact point with the dentin at right angles. The testing device moved with a defined speed of $1 \mathrm{~mm} / \mathrm{min}$ toward the plunger. The shear bond strength that needs to separate the test materials from the dentin surface was calculated with a special software program (Blue heal 3). 


\section{Microleakage}

Twenty (noncarious and nonrestored) human maxillary premolars were selected for the current study. Two class II box-only cavities with buccolingual width of $3.0 \mathrm{~mm}$, an occlusogingival height of approximately $6.0 \mathrm{~mm}$, and an axial depth of $2 \mathrm{~mm}$ at the cervical floor were prepared on the mesial and distal surfaces of each tooth. The proximal box margins were placed $1.0 \mathrm{~mm}$ below the cementoenamel junction. All the teeth were finished and polished using composite finishing bur 7901 and soflex disks. The specimens were subjected to 1000 thermocycles comprising of 30 seconds at $5^{\circ} \mathrm{C} \pm 2^{\circ} \mathrm{C}$ and 30 seconds at $55^{\circ} \mathrm{C} \pm 2^{\circ} \mathrm{C}^{8,9}$

Teeth were then impermeabilized using sticky wax. Entire teeth were painted with nail varnish except $1 \mathrm{~mm}$ from restoration margin. Teeth were then placed at room temperature for 12 hours dipped in $5 \%$ methylene blue dye. The teeth on removal from the dye solution were superficially cleaned with pumice slurry and rubber cup. The teeth specimens were then sectioned longitudinally with double-sided diamond disk. Two sections were made from each tooth. Dye penetration extent was studied under digital microscope (Hirox Digital Scope, Japan) at 50× magnification. The part of sectioned tooth showing highest amount of microleakage was scored. ${ }^{10}$

Microleakage was scored using the following criteria 8 :

- No leakage

- Partial dye penetration

- Penetration of dye along the gingival wall which does not include axial wall

- Penetration of dye along the axial wall.

Biodentine was mixed with the liquid and put in an amalgamator for 30 seconds. Mixed biodentine was placed in the cavity and excess material was removed before the setting time of 12 minutes. Surefil SDR was placed after conditioning the tooth with $37 \%$ phosphoric acid and adhesive (Tetric N Bond, Ivoclar Vivadent) and light cured (Bluephase G2, Ivoclar Vivadent). EverX posterior was placed after conditioning the tooth surface with GC aenial, a total etch component and light cured (Bluephase G2, Ivoclar Vivadent). Activa Bioactive Restorative was placed in the cavity after conditioning the tooth surface with $37 \%$ phosphoric acid and light cured (Bluephase G2, Ivoclar Vivadent).

\section{Statistical Analysis}

Statistical analysis was done using Statistical Package for the Social Sciences (SPSS) 19.0 (v.19.0, IBM, Chicago). Mean and standard deviation from the recorded values of shear bond strength were calculated. Intergroup comparison was done with one-way analysis of variance (ANOVA) followed by pairwise comparison using Tukey honestly significant difference (HSD) post hoc test. Samples showing microleakage at various sites for the study groups were counted and expressed in percentage.

\section{RESULTS}

\section{Shear Bond Strength}

Mean and standard deviation from the recorded values of shear bond strength were calculated. Table 1 and Graph 1 show the mean shear bond strength of the test materials. Intergroup comparison was done with one-way ANOVA followed by Tukey HSD post hoc test. Table 2 shows the comparison of the mean shear bond strength of the test materials using one-way ANOVA and pairwise comparison using Tukey HSD post hoc test. Biodentine

Table 1: Mean shear bond strength of the test materials

\begin{tabular}{llll}
\hline Study groups & $n$ & Mean (in MPa) & Std. Dev. \\
\hline Biodentine & 10 & 2.33 & 0.230 \\
EverX posterior & 10 & 8.87 & 0.148 \\
Bioactive Restorative & 10 & 6.28 & 0.157 \\
SDR & 10 & 10.12 & 0.457 \\
\hline
\end{tabular}

Table 2: Comparison of the mean shear bond strength of the test materials using one-way ANOVA and pairwise comparison using Tukey HSD post hoc test

\begin{tabular}{llllll}
\hline Source of variation & Sum of squares & d.f. & Variance & $F$ & -value \\
\hline Between groups & 361.0428 & 3 & 120.3476 & 186.7157 & $0.000^{*}$ \\
Within group & 23.2038 & 36 & 0.6446 & & \\
Total & 384.2466 & 39 & & \\
Tukey HSD post hoc test & & & &
\end{tabular}

\begin{tabular}{|c|c|c|c|c|}
\hline & \multirow[b]{2}{*}{ Mean difference } & \multicolumn{2}{|c|}{$95 \%$ confidence interval } & \multirow[b]{2}{*}{$p$-value } \\
\hline & & Upper & Lower & \\
\hline Group I vs group II & 6.45 & 7.5070 & 5.5730 & $0.0000^{*}$ \\
\hline Group I vs group III & 3.95 & 4.9170 & 2.9830 & $0.0000^{*}$ \\
\hline Group I vs group IV & 7.88 & 8.8470 & 6.9130 & $0.0203^{*}$ \\
\hline Group II vs group III & 2.59 & -1.6230 & -3.5570 & $0.0000^{*}$ \\
\hline Group II vs group IV & 1.34 & 2.3070 & 0.3730 & $0.0035^{*}$ \\
\hline Group III vs group IV & 3.93 & 4.8970 & 2.9630 & $0.0000^{*}$ \\
\hline
\end{tabular}

*significant at $95 \%$ confidence interval 


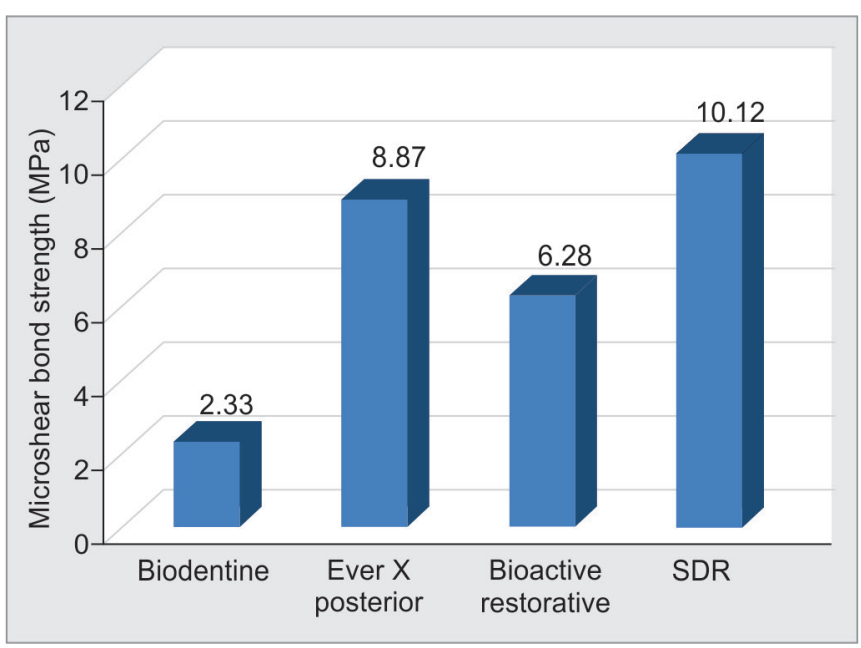

Graph 1: Mean shear bond strength of the test materials

Table 3: Samples showing microleakage at various sites for the study groups

\begin{tabular}{lllll}
\hline & \multicolumn{4}{c}{ Number of Samples (\%) } \\
\cline { 2 - 5 } Microleakage & \multicolumn{4}{c}{ EverX } \\
\hline No leakage & $3(30)$ & 0 & $4(40)$ & $4(40)$ \\
Cervical & $2(20)$ & $6(60)$ & $4(40)$ & $5(50)$ \\
Marginal & $1(10)$ & $1(10)$ & $1(10)$ & 0 \\
Cervical and marginal & $4(40)$ & $3(30)$ & $1(10)$ & $1(10)$ \\
\hline
\end{tabular}

showed the lowest shear bond strength of all the tested materials. SDR surefil showed the highest bond strength followed by EverX and Activa Bioactive Restorative $(\mathrm{F}=186.7157, \mathrm{p}<0.05)$.

\section{Microleakage}

Analysis showed microleakage score highest for Biodentine. SDR surefil showed better properties of microleakage followed by EverX and Activa Bioactive Restorative. Marginal leakage was observed at the margins, occlusal and gingival levels. Table 3 and Graph 2 show samples showing microleakage at various sites for the study groups.

\section{DISCUSSION}

A good dental cement used as repair material should possess chemical binding, easy handling characteristics, minimal marginal breakdown and minimal polymerization shrinkage, high resistance to wear, high cohesive strength, and good color stability. ${ }^{11}$ During photopolymerization, monomers form a polymer network and resin-based composites become solid and shrink. ${ }^{12,13}$ Shrinkage manifests itself as stress at the bonded cavity walls, which may develop interfacial defects, enamel fracture, cuspal movements, and microcracks. In order to reduce polymerization shrinkage stress and to obtain

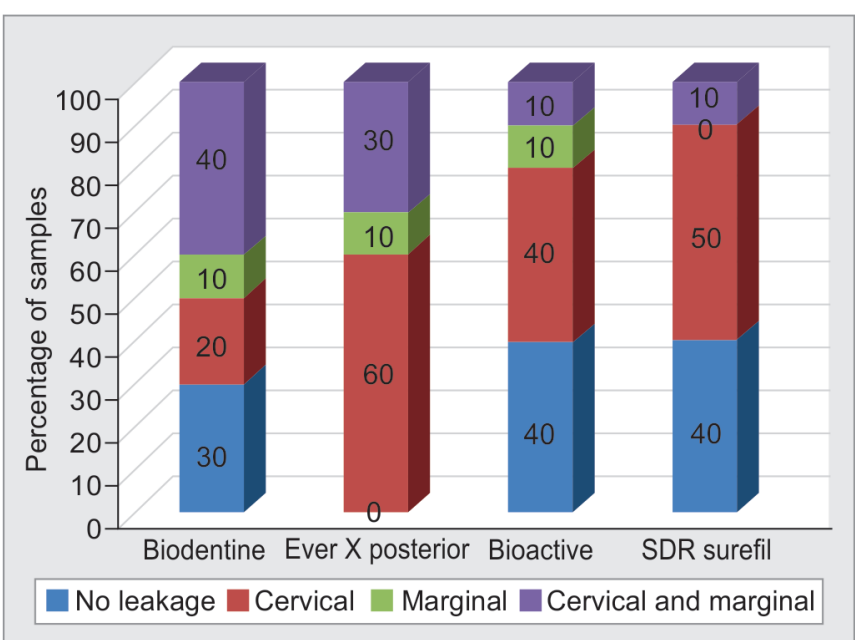

Graph 2: Samples showing microleakage at various sites for the study groups

optimal outcomes, composites need to be placed in increments of $2 \mathrm{~mm} .{ }^{14,15}$ The incremental layering technique promotes a smaller ratio of the areas of bonded to unbounded composite resin layer achieving a lower $\mathrm{C}$ factor during polymerization of each layer. An increment thickness of $2 \mathrm{~mm}$ or less provides sufficient light penetration for polymerization resulting in enhanced physical and mechanical properties with improved marginal adaptations. A higher degree of conversion of the composites resin also contributes to decreased cytotoxicity. ${ }^{16,17}$

Clinical studies show that fracture of restoration is the most common reason for failure of indirectly or directly made posterior composite restorations. Use of material combinations has shown substantial improvements in load-bearing capacity of restorations. The short fiber composite resin has revealed control of polymerization shrinkage stress by fiber reorientation and thus reducing the marginal microleakage compared with conventional particulate filler composite resin. ${ }^{18}$ The bulk short fiber supports the particulate filler composite layer and helps in prevention of cracks in composite restoration. The fiber takes up the stress from the polymer matrix and thus reinforces the polymer. The following can be achieved when the fibers have length equal to or greater than that of the critical fibers. ${ }^{19}$ Poor adhesion can be compensated by mechanical friction of fibers to polymer matrix at the interface. $^{20}$

GC EverX Posterior is a fiber-reinforced composite designed to be used as dentin replacement. According to the manufacturers, the short fibers of GC EverX Posterior make it a good substructure for the reinforcement of large-sized composite restorations. Fibers prevent and stop crack propagation through the restoration, thereby preventing composite failure. ${ }^{21}$

In surefil, SDR increase in polymerization stress is reduced with time, which is due to SDR-patented urethane dimethacrylate structure in this composite. ${ }^{22}$ 
Urethane with incorporated photoactive group is able to control the polymerization kinetics. In bulk-fill placement technique, SDR has better internal adaptation than conventional composites in high $\mathrm{C}$ factor cavities. ${ }^{16}$

Biodentine was introduced by Septodont research group. It is a calcium silicate-based material based on Active Biosilicate Technology designed to treat damaged dentin. ${ }^{23}$ When compared with ProRoot MTA and MTA Plus infurcation perforation repairs, it was found that MTA had less bond strength than Biodentine. ${ }^{24}$

Microleakage is defined as clinically undetectable passage of bacteria, fluids, molecules, or ions between a cavity wall and the restorative material. ${ }^{25}$ Microleakage as an assessment parameter for restoration failure remains controversial. But, microleakage assessment is still the most frequently used test method for a new material or combination of materials. ${ }^{26}$ Many studies that have employed similar techniques for the same material have often provided contradictory results. This contrast may have resulted from different handling and manipulation of materials. ${ }^{25}$ Dye penetration technique is a commonly used, simple, and comparable method for microleakage evaluation; hence, it was utilized in the present study.

Activa Bioactive Restorative is a highly esthetic, bioactive composite that delivers all the advantages of glass ionomers in a strong, resilient, resin matrix that will not chip or crumble. It chemically bonds to teeth, seals against bacterial microleakage, releases more fluoride, and is more bioactive than glass ionomers, and is more durable and fracture resistant than composites. Activa Restorative contains glass particles and polyacid components of resin-modified glass ionomer cements, which undergo the acid/base neutralization hardening reaction of all glass-ionomer systems. In addition, they also contain a bioactive ionic resin matrix, and thereby are able to achieve polymerization by both light cure and chemical cure. Thus, there are three hardening mechanisms involved with the Activa Restorative. ${ }^{7,13}$ Activa products are unique and first of its kind as their physical properties are comparable to those of the resin-based composites tested, along with biological properties that rival glassionomer systems. ${ }^{27,28}$ Other advantages include easy handling like most injectable resin-based composites and no need of bonding agent when repairing primary teeth. The material is left undisturbed for about 20 seconds after injection, which allows the polyacid component to etch the tooth. Also, retention achieved with adhesive bonding is augmented by mechanical undercutting as is the case in most cavity preparations. "White line" margins, which are considered typical of freshly finished bonded resinbonded composite restorations, are prominently absent, which is most likely owing to low polymerization shrinkage. For permanent tooth repair, standard acid-etching protocol or self-etching bonding agent can be utilized. In event of the light-beam penetration not being ideal, polymerization is achieved by chemical cure and the acid/base neutralization hardening reaction of the glassionomer components. ${ }^{29}$

\section{CONCLUSION}

In this study, SDR (surefil) showed better shear bond strength and showed better microleakage quality as urethane with incorporated photoactive groups is able to control the polymerization kinetics. Bulk-fill short fiber-reinforced composite showed similar properties of shear bond strength and microleakage with SDR. Activa Bioactive Restorative properties were not that comparable with SDR (surefil), and biodentine showed the least qualities. The present study showed that flowable and fiber-reinforced composites have better properties.

\section{REFERENCES}

1. Raju VG, Venumbaka NR, Mungara J, Vijayakumar P, Rajendran S, Elangovan A. Comparative evaluation of shear bond strength and microleakage of tricalcium silicatebased restorative material and radioopaque posterior glass ionomer restorative cement in primary and permanent teeth: an in vitro study. J Indian Soc Pedod Prev Dent 2014 Oct-Dec;32(4):304-310.

2. Cramer NB, Stansbury JW, Bowman CN. Recent advances and developments in composite dental restorative materials. J Dent Res 2011 Apr;90(4):402-416.

3. Demarco FF, Corrêa MB, Cenci MS, Moraes RR, Opdam NJ. Longevity of posterior composite restorations: not only a matter of materials. Dent Mater 2012 Jan;28(1):87-101.

4. Walter R. Bulk-fill flowable composite resins. J Esthet Restor Dent 2013 Feb;25(1):72-76.

5. Garoushi S, Säilynoja E, Vallittu PK, Lassila L. Physical properties and depth of cure of a new short fiber reinforced composite. Dent Mater 2013 Aug;29(8):835-841.

6. Laurent $\mathrm{P}$, Camps J, De Méo M, Déjou J, About I. Induction of specific cell responses to a $\mathrm{Ca} 3 \mathrm{SiO}$ 5-based posterior restorative material. Dent Mater 2008 Nov;24(11):1486-1494.

7. The future of dentistry now in your hands. PULPDENT ${ }^{\circledR}$ publication. Watertown, MA: Pulpdent Corporation; 2014.

8. Rekha CV, Varma B. Comparative evaluation of tensile bond strength and microleakage of conventional glass ionomer cement, resin modified glass ionomer cement and compomer: an in vitro study. Contemp Clin Dent 2012 Jul;3(3):282.

9. Gonzalez NA, Abu Kasim NH, Aziz RD. Microleakage testing. Ann Dent 1997;4(1):1-54.

10. Masih S, Thomas AM, Koshy G, Joshi JL. Comparative evaluation of the microleakage of two modified glass ionomer cements on primary molars. An in vivo study. J Indian Soc Pedod Prev Dent 2011 Apr;29(2):135.

11. Croll TP, Berg JH. Nano-ionomer restorative cement. Inside Dent 2009;5(1):60-67.

12. Versluis AB, Douglas WH, Cross MB, Sakaguchi RL. Does an incremental filling technique reduce polymerization shrinkage stresses? J Dent Res 1996 Mar;75(3):871-878. 
13. van Dijken JW, Pallesen U. A randomized controlled three year evaluation of "bulk-filled" posterior resin restorations based on stress decreasing resin technology. Dent Mater 2014 Sep;30(9):e245-e251.

14. Lazarchik DA, Hammond BD, Sikes CL, Looney SW, Rueggeberg FA. Hardness comparison of bulk-filled/transtooth and incremental-filled/occlusally irradiated composite resins. J Prosthet Dent 2007 Aug;98(2):129-140.

15. Van Ende A, De Munck J, Van Landuyt KL, Poitevin A, Peumans M, Van Meerbeek B. Bulk-filling of high C-factor posterior cavities: effect on adhesion to cavity-bottom dentin. Dent Mater 2013 Mar;29(3):269-277.

16. Park J, Chang J, Ferracane J, Lee IB. How should composite be layered to reduce shrinkage stress: incremental or bulk filling? Dent Mater 2008 Nov;24(11):1501-1505.

17. Poskus LT, Placido E, Cardoso PE. Influence of placement techniques on Vickers and Knoop hardness of class II composite resin restorations. Dent Mater 2004 Oct 31;20(8):726-732.

18. Garoushi S, Vallittu PK, Lassila LV. Direct restoration of severely damaged incisors using short fiber-reinforced composite resin. J Dent 2007 Sep;35(9):731-736.

19. van Dijken JW, Sunnegårdh-Grönberg K. Fiber-reinforced packable resin composites in class II cavities. J Dent 2006 Nov;34(10):763-769.

20. Cheng TH, Jones FR, Wang D. Effect of fibre conditioning on the interfacial shear strength of glass-fibre composites. Composit Sci Technol 1993 Dec;48(1):89-96.
21. Available from: http://www.gceurope.com/products/detail. php?id=162 [cited 2016 Jul 8].

22. Reis A. Evaluation of a novel composite restorative system for posterior teeth: microleakage, bond strength, and gap formation on Class II MOD cavities. Data on File. 2009. Available from: www.surefilsdrflow.com.23.

23. Jain P, D Raj Ja. Dentin substitutes: a review. Int J Pharm Bio Sci 2015 July;6(3):383-391.

24. Aggarwal V, Singla M, Miglani S, Kohli S. Comparative evaluation of push-out bond strength of ProRoot MTA, Biodentine, and MTA Plus in furcation perforation repair. J Conserv Dent 2013 Sep;16(5):462-465.

25. Kidd EA. Microleakage: a review. J Dent 1976 Sep;4(5): 199-206.

26. Heintze SD. Systematic reviews: I. The correlation between laboratory tests on marginal quality and bond strength. II. The correlation between marginal quality and clinical outcome. J Adhes Dent 2007 Jan;9 (Suppl) 1:77.

27. Burgess JO, Norling BK, Rawls HR, Ong JL. Directly placed esthetic restorative materials - the continuum. Compend Contin Educ Dent 1996 Aug;17(8):731-732.

28. Berg JH. The continuum of restorative materials in pediatric dentistry-a review for the clinician. Pediatr Dent 1998 Mar;20(2):93-100.

29. Croll TP, Berg JH, Donly KJ. Dental repair material: a resinmodified glass-ionomer bioactive ionic resin-based composite. Compend Contin Educ Dent 2015 Jan;36(1):60-65. 